\title{
$\checkmark$ Research Square

\section{Ultrafast imaging of spontaneous symmetry breaking in a photoionized molecular system}

\section{Min Li}

Huazhong University of Science and Technology https://orcid.org/0000-0001-7790-9739

\section{Ming Zhang}

Peking University

Oriol Vendrell

University of Heidelberg https://orcid.org/0000-0003-4629-414X

\section{Zhenning Guo}

Peking University

\section{Qianru Zhu}

Huazhong University of Science and Technology

\section{Xiang Gao}

Huazhong University of Science and Technology

\section{Lushuai Cao}

Huazhong University of Science and Technology

\section{Keyu Guo}

Huazhong University of Science and Technology

\section{Qinqin Su}

Huazhong University of Science and Technology

\section{Wei Cao}

Huazhong University of Science and Technology

\section{Siqiang Luo}

Huazhong University of Science and Technology

\section{Jiaqing Yan}

Huazhong University of Science and Technology

\section{Yueming Zhou}

Huazhong University of Science and Technology

\section{Yunquan Liu}

State Key Laboratory for Mesoscopic Physics and Frontiers Science Center for Nano-optoelectronics, School of Physics, School of Physics, Peking University

\section{Peixiang Lu}

Huazhong University of Science and Technology https://orcid.org/0000-0001-6993-8986

\section{Zheng Li ( $\square$ zheng.li@pku.edu.cn )}

Peking University https://orcid.org/0000-0002-0329-1275 


\section{Article}

Keywords: Jahn-Teller effect, Jahn-Teller symmetry, ultrafast ion-coincidence Coulomb explosion imaging, Jahn-Teller deformations, ultrafast dynamics

Posted Date: August 24th, 2020

DOl: https://doi.org/10.21203/rs.3.rs-56501/v1

License: (c) (1) This work is licensed under a Creative Commons Attribution 4.0 International License. Read Full License

Version of Record: A version of this preprint was published at Nature Communications on July 9th, 2021. See the published version at https://doi.org/10.1038/s41467-021-24309-z. 


\title{
Ultrafast imaging of spontaneous symmetry breaking in a photoionized molecular system
}

\author{
Min Li* ${ }^{1}$ Ming Zhang* ${ }^{2,3}$ Oriol Vendrell, ${ }^{4}$ Zhenning Guo, ${ }^{2}$ Qianru Zhu, ${ }^{5}$ Xiang \\ Gao, ${ }^{5}$ Lushuai Cao, ${ }^{5}$ Keyu Guo, ${ }^{1}$ Qin-Qin $\mathrm{Su},{ }^{1}$ Wei Cao,, * Siqiang Luo, ${ }^{1}$ Jiaqing \\ Yan, ${ }^{1}$ Yueming Zhou, ${ }^{1}$ Yunquan Liu, ${ }^{2,}$ † Zheng $\mathrm{Li},{ }^{2}$, 周 and Peixiang $\mathrm{Lu}^{1,6,7,\{}$ \\ ${ }^{1}$ Wuhan National Laboratory for Optoelectronics and School of Physics, \\ Huazhong University of Science and Technology, Wuhan 430074, China \\ ${ }^{2}$ State Key Laboratory for Mesoscopic Physics and \\ Collaborative Innovation Center of Quantum Matter, \\ School of Physics, Peking University, Beijing 10087, China \\ ${ }^{3}$ University of Science and Technology Beijing, Beijing 1008\%, China \\ ${ }^{4}$ Physikalisch-Chemisches Institut, Universität Heidelberg, \\ Im Neuenheimer Feld 229, D-69120 Heidelberg, Germany \\ ${ }^{5}$ MOE Key Laboratory of Fundamental Physical Quantities Measurement and Hubei \\ Key Laboratory of Gravitation and Quantum Physics, PGMF and School of Physics, \\ Huazhong University of Science and Technology, Wuhan 430074, China \\ ${ }^{6}$ Hubei Key Laboratory of Optical Information and Pattern Recognition, \\ Wuhan Institute of Technology, Wuhan 430205, China \\ ${ }^{7}$ CAS Center for Excellence in Ultra-intense Laser Science, Shanghai 201800, China
} (Dated: July 31, 2020) 


\begin{abstract}
The Jahn-Teller effect is an essential mechanism of spontaneous symmetry breaking in molecular and solid state systems, and has far-reaching consequences in many fields. Up to now, to direct image the onset of Jahn-Teller symmetry breaking remains unreached. Employing ultrafast ioncoincidence Coulomb explosion imaging with sub-10 fs resolution, we unambiguously imaged the ultrafast dynamics of Jahn-Teller deformations of $\mathrm{CH}_{4}^{+}$cation in real space. It is unraveled that the Jahn-Teller deformation from $C_{3 v}$ to $C_{2 v}$ geometries takes a characteristic time of about 20 femtoseconds for this system. Classical and quantum molecular dynamics simulations agree well with the measurement, and reveal dynamics for the build-up of the $C_{2 v}$ structure involving complex phasing process of multiple vibrational pathways of the $\mathrm{CH}_{4}^{+}$cation.
\end{abstract}

$1 \quad$ PACS numbers: $32.80 . \mathrm{Fb}, 42.50 . \mathrm{Hz}, 32.80 . \mathrm{Rm}$ 
In the 1930s, Hermann Jahn and Edward Teller discovered a now celebrated theorem demonstrating the intrinsic geometric instability of degenerate electronic states based on group theory [1]. This instability leads to spontaneous symmetry breaking of the molecular structure, known as Jahn-Teller (JT) effect, which removes the degeneracy of the electronic state and lowers the overall energy of the molecules. Essentially, the JT effect originates from the coupling of electronic and nuclear degrees of freedom (DOF) in molecules, and heralds the breakdown of the Born-Oppenheimer approximation [2], which is the basis for much of our understanding of molecular structure and dynamics. The JT effect is a widespread phenomenon occurring in a broad range of molecules, transition-metal complexes, and solids. From the fundamental physics perspective, the JT effect is a concrete example of spontaneous symmetry breaking, which has far-reaching consequences in quantum field theory and the Standard Model of particle physics, such as the Higgs mechanism [3].

Structural properties appearing as a consequence of the JT effect have been extensively demonstrated in static measurements with high-resolution optical spectroscopy [46], electron paramagnetic resonance spectroscopy [7, 8], rotationally resolved pulsed-fieldionization zero-kinetic-energy photoelectron spectra [9 14, as well as Coulomb explosion experiments [15, 16]. However, accessing the short-time structural dynamics caused by the JT effect, i.e. the onset of symmetry breaking from an initially symmetric configuration, remains a great challenge, and a direct experimental imaging of structural symmetry breaking in real space has not yet been realized [17]. Accessing these structural dynamics is key to understanding the early stages of photo-triggered chemical bond cleavage and molecular dynamics leading to energy and charge transfer processes [18]. In this connection, recent ground-breaking transient absorption spectroscopy measurements in crystals [19] and molecules [20] report time-dependent energy level shifts immediately after the interaction with a pump pulse, which can be traced back to the onset of JT distortions. However a comprehensive, real-space picture of these ultrafast processes is still missing.

In this work, we propose and demonstrate a scheme to experimentally image the dynamics of the JT distortion and apply it to the photoionized methane cation. The methane cation is one of the simplest floppy systems exhibiting JT distortions in its triply degenerate ground state. The topology of the corresponding potential energy surfaces is well known [21] and constitutes the basis for our analysis. A schematic of the dynamics unfolding after a strong pump laser pulse is illustrated in Fig. 1. The molecular geometry can be detected by Coulomb 


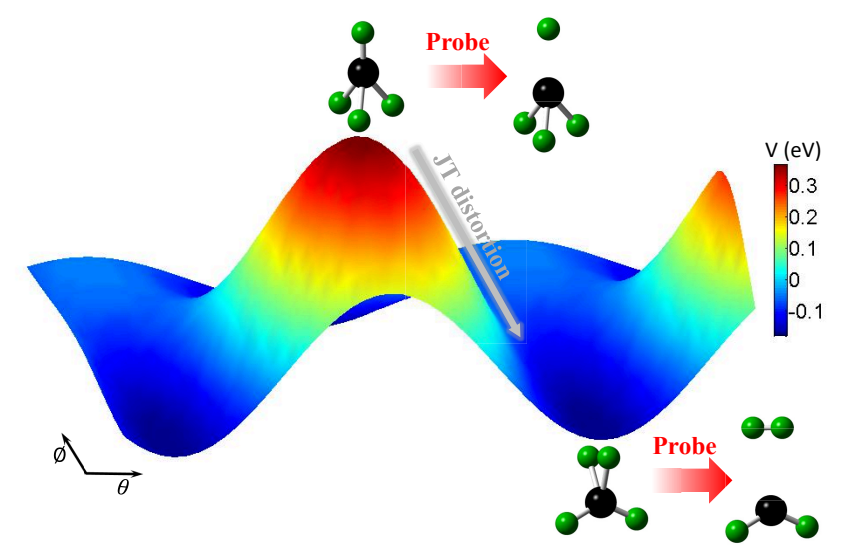

FIG. 1. Scheme of probing JT deformation in a photoionized $\mathrm{CH}_{4}^{+}$cation. The $\mathrm{CH}_{4}^{+}$ cation in a $C_{3 v}$ geometry (at the peak of the potential energy surface) undergoes JT distortion to a $C_{2 v}$ geometry (at the bottom of the potential energy surface). By recording the coincident fragments from Coulomb explosion of those geometries as a function of time between the pumpprobe laser pulses, the dynamics of the JT deformation can be revealed.

1 explosion imaging under the influence of a subsequent probe laser pulse. By simultaneously recording the kinetic energies and momenta of the fragments from two- and three-body Coulomb explosion as a function of the pump-probe time delay, the dynamics of the JT distortion is mapped in time and real space.

\section{Experimental Results}

A strong pump laser pulse photoionizes $\mathrm{CH}_{4}$ and populates the $\mathrm{CH}_{4}^{+}$in its ground ${ }^{2} \mathrm{~F}$ state with $T_{d}$ symmetry, which subsequently deforms owing to the JT effect. In Fig. 2, the measured kinetic energy release (KER) distributions are plotted as a function of the time delay between the two 25-fs pulses for the two-body breakup channels of $\mathrm{CH}_{3}^{+}+\mathrm{H}^{+}$ and $\mathrm{CH}_{2}^{+}+\mathrm{H}_{2}^{+}$. In order to separate Coulomb explosion events from other dissociative fragmention channels, only those events are shown where two ions have been detected in coincidence and fulfill the momentum conservation condition. One can see that the timeintegrated KER are nearly the same for those two breakup channels. Both of them reveal the maximal yield at $\sim 5 \mathrm{eV}$.

Zero time delay in Fig. 2 means that the pump and probe pulses come at the same time. One sees that the KER spectra are dominated by time-independent features at $\mathrm{KER} \sim 5 \mathrm{eV}$ 

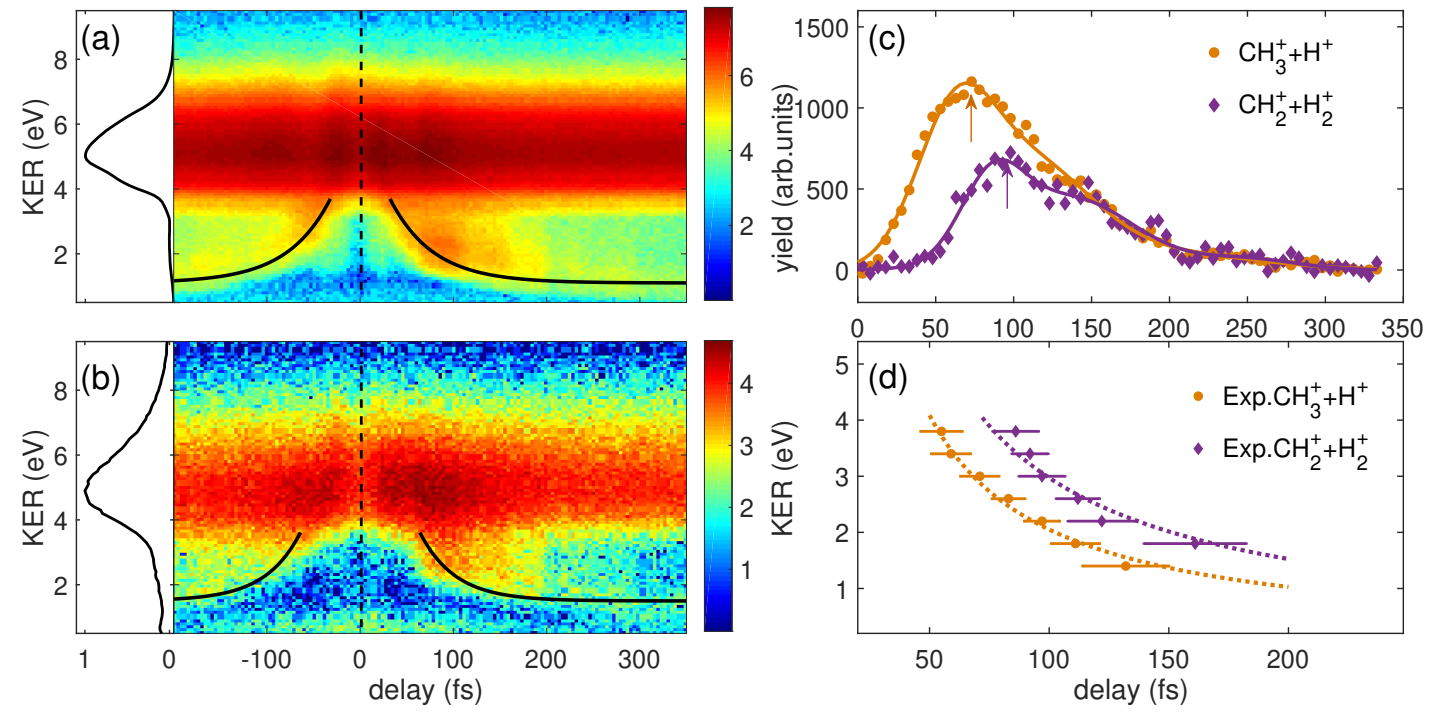

FIG. 2. Time evolution of two-body breakup channel. (a)(b) The measured ion yields with respect to the KER and the pump-probe time delay for the pathways of $\mathrm{CH}_{3}^{+}+\mathrm{H}^{+}$(a) and $\mathrm{CH}_{2}^{+}+\mathrm{H}_{2}^{+}(\mathrm{b})$. The time-integrated KER distributions are shown in the left panels. The black solid curves in (a) and (b) are used to guide the time-dependent feature. (c) The measured ion yields with respect to the time delay at the KER of $3.0 \mathrm{eV}$ for the pathways of $\mathrm{CH}_{3}^{+}+\mathrm{H}^{+}$ and $\mathrm{CH}_{2}^{+}+\mathrm{H}_{2}^{+}$(multiplied by a factor of 5 for visual convenience). The arrows show the peaks of the time-dependent distributions. (d) The peak extracted from the measured time-dependent distribution for different KERs. The dotted lines are the fits of the experimental data.

caused by the interaction with only one of the laser pulses [22]. The KER spectra also display some time-dependent features that reflect the behavior of parts of the nuclear wave packet, as guided by the black solid lines. Those time-dependent features are symmetric about zero delay for both $\mathrm{CH}_{2}^{+}+\mathrm{H}_{2}^{+}$and $\mathrm{CH}_{3}^{+}+\mathrm{H}^{+}$channels. Interestingly, the time-dependent feature of the $\mathrm{CH}_{2}^{+}+\mathrm{H}_{2}^{+}$channel appears later than that of the $\mathrm{CH}_{3}^{+}+\mathrm{H}^{+}$channel for the positive delay. To show this phenomenon clearer, we take line-outs at the KER of 3.0 eV from Figs. 2(a) and 2(b), which are shown in Fig.2(c). By fitting the time-dependent distributions, we find that the maximum of the time-dependent yields of the $\mathrm{CH}_{2}^{+}+\mathrm{H}_{2}^{+}$ breakup channel appears $\sim 20$ fs later than that of the $\mathrm{CH}_{3}^{+}+\mathrm{H}^{+}$breakup channel at the KER of $3.0 \mathrm{eV}$. Figure 2(d) shows the maxima of the time-dependent yields as a function of the KER for the two breakup channels. One can see that for all KERs the $\mathrm{CH}_{2}^{+}+\mathrm{H}_{2}^{+}$ 
channel appears $\sim 20$ fs later than the $\mathrm{CH}_{3}^{+}+\mathrm{H}^{+}$channel.

To image the molecular dynamics in real time and space, we further measured the threebody Coulomb explosion channel $\left(\mathrm{CH}_{2}^{+}+\mathrm{H}^{+}+\mathrm{H}^{+}\right)$, which could provide detailed information about the bond angles. In Fig. 3(a)-(d), we show the Newton plots of the three-body breakup channel $\left(\mathrm{CH}_{2}^{+}+\mathrm{H}^{+}+\mathrm{H}^{+}\right)$at different time delays. Here we selected the events with the same final momenta for the two $\mathrm{H}^{+}$, which implies similar $\mathrm{C}-\mathrm{H}$ bond length at the instant of Coulomb explosion. It is immediately clear that there are several spot-like structures in the Newton plot (labelled as $S 1, S 2$, and $S 3$ in Fig. 3(b)), which corresponds to different sets of bond angles, they directly reflect distinctive symmetric configurations at the instant of Coulomb explosion.

\section{Discussions}

To interpret the experimental data, we employ molecular dynamics (MD) method to simulate the $\mathrm{CH}_{4}^{+}$symmetry breaking and to understand the unexpected long time-delay of $\sim 20$ fs between the measured peak yields of $\mathrm{CH}_{3}^{+}+\mathrm{H}^{+}$and $\mathrm{CH}_{2}^{+}+\mathrm{H}_{2}^{+}$fragmentation channels. Fig. 4 illustrates how the simulated $\mathrm{CH}_{3}^{+}+\mathrm{H}^{+}$and $\mathrm{CH}_{2}^{+}+\mathrm{H}_{2}^{+}$yields peak at 16 fs and $29 \mathrm{fs}$, which is in qualitative agreement with the time difference from the two-body Coulomb explosion measurement. The peak time difference, which is essentially longer than the quarter of a period of any vibrational mode, strongly implies complex dynamics involving multiple driving modes.

The peak time difference between the $\mathrm{CH}_{3}^{+}+\mathrm{H}^{+}$and $\mathrm{CH}_{2}^{+}+\mathrm{H}_{2}^{+}$channels of the two-body Coulomb explosion can be understood as follows. In the $\mathrm{CH}_{4}^{+}$cation, both $f_{2}$ bending mode and $f_{2}$ stretching mode lead to the $C_{2 v}$ structures of the lowest energy [21]. The $f_{2}$ bending mode enables relaxation from the ${ }^{2} F$ state to the ${ }^{2} E$ state of $C_{3 v}$ configurations. The $C_{3 v}$ geometry of the $\mathrm{CH}_{4}^{+}$cation has one short and three long $\mathrm{C}-\mathrm{H}$ bonds, which correlates with the $\mathrm{CH}_{3}^{+}+\mathrm{H}^{+}$breakup channel in the Coulomb explosion caused by the probe pulse (at the peak of the potential energy surface of Fig. 1). The $C_{3 v}$ geometry is the apex of the double cone with surrounding $C_{2 v}$ minima. In the $C_{3 v}$ geometry, the cation encounters again a doubly degenerate electronic state, which further relaxes to lower symmetry via JT distortions [21]. Linear combination of symmetric modes, such as $f_{2}$ stretching and bending, subsequently leads the cation in $C_{3 v}$ geometry to land on the ${ }^{2} B_{2}$ state of the lowest 


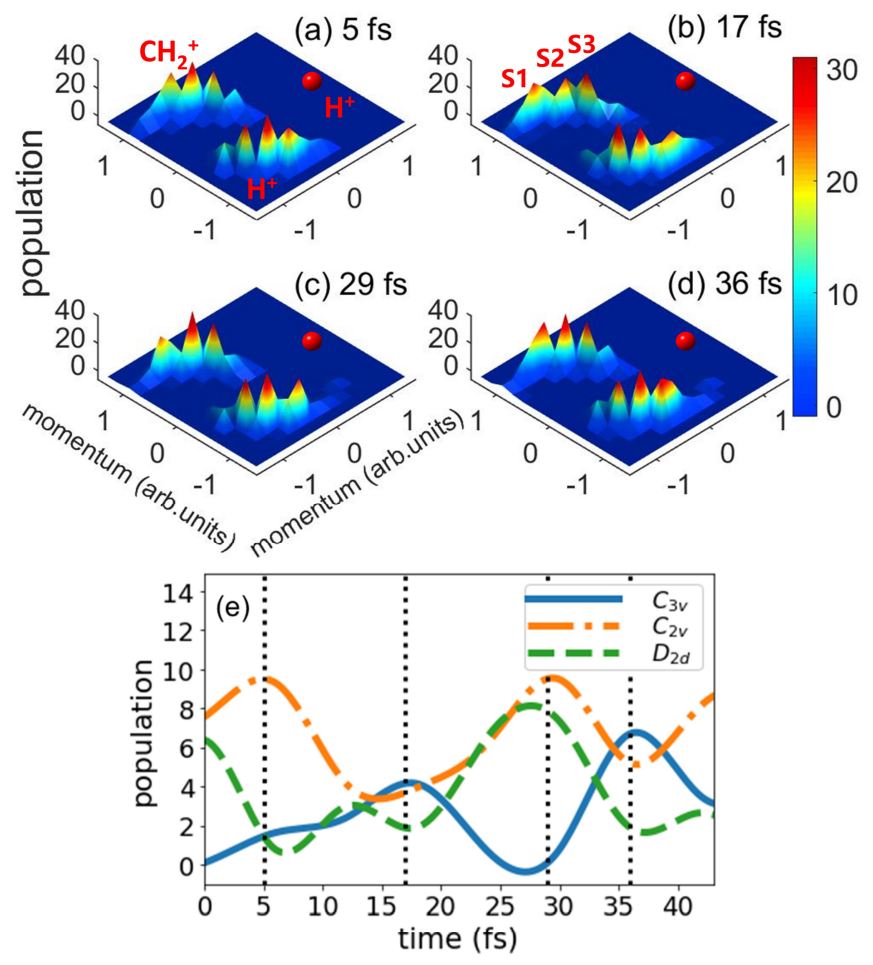

FIG. 3. Time evolution of three-body breakup channel. (a)-(d) Newton plot at the delay of $5 \mathrm{fs}, 17 \mathrm{fs}, 29 \mathrm{fs}$, and 36 fs. Several spot-like structures in the Newton plot are indicated by S1, S2, and S3 in (b), which represent molecular geometries of different symmetry types at the instant of Coulomb explosion. The momentum vector of the first $\mathrm{H}^{+}$is set as one unit at $(0,1)$, as shown by the red sphere. The momentum vectors of the $\mathrm{CH}_{2}^{+}$ion and the second $\mathrm{H}^{+}$ion are normalized with respect to the length of momentum vector of the first $\mathrm{H}^{+}$ion. (e) The experimentally extracted evolution of $C_{3 v}$ like, $C_{2 v}$ like and $D_{2 d}$ like geometries. The experimental data are obtained by selecting those events with the same final momenta for two $\mathrm{H}^{+}$.

${ }_{1} C_{2 v}$ symmetry (at the bottom of the potential energy surface in Fig. 1). In the $C_{2 v}$ symmetric $2 \mathrm{CH}_{4}^{+}$, two long $\mathrm{C}-\mathrm{H}$ bonds are separated by a small angle $\left(56.3^{\circ}\right)$ while two short $\mathrm{C}-\mathrm{H}$ bonds 3 are separated by a large angle $\left(121.9^{\circ}\right)$. The closest $\mathrm{H}-\mathrm{H}$ distance in the $C_{2 v}$ geometry is $41.03 \AA$ [21], which is nearly the same as the equilibrium internuclear distance of $\mathrm{H}_{2}^{+}(1.06 \AA)$. 5 Similar to the neutral excited $\mathrm{CH}_{4}$ molecule in the degenerate ${ }^{1} F_{2}$ state [23], the deformation ${ }_{6}$ to $C_{2 v}$ via $C_{3 v}$ symmetric geometry in the ${ }^{2} B_{2}$ cationic state finally provides the breakup 7 channel $\mathrm{CH}_{2}^{+}\left({ }^{1} A_{1}\right)+\mathrm{H}_{2}^{+}\left({ }^{2} \Sigma_{g}^{+}\right)$upon double ionization by the probe laser pulse. Thus the s time difference between the $\mathrm{CH}_{3}^{+}+\mathrm{H}^{+}$and $\mathrm{CH}_{2}^{+}+\mathrm{H}_{2}^{+}$channels in Fig. 2 is equivalent to the 
(a)

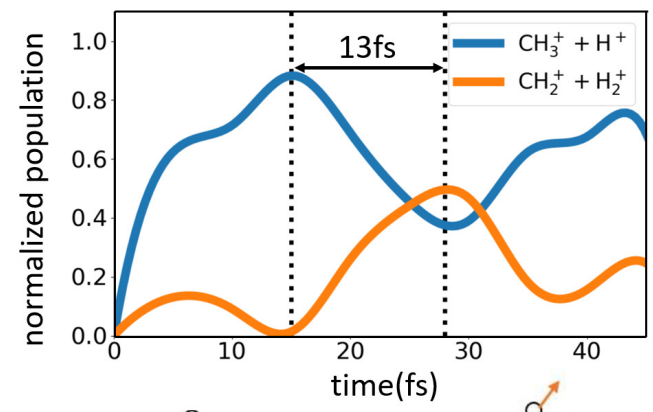

(b)

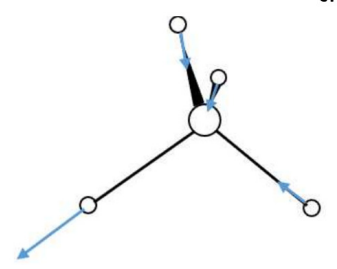

(c)

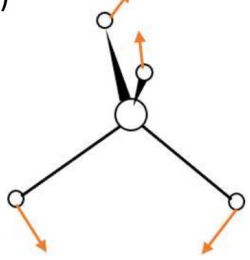

(d)

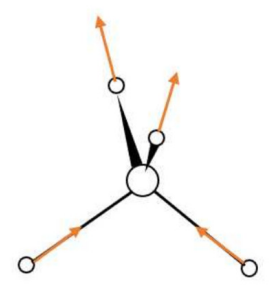

(e)

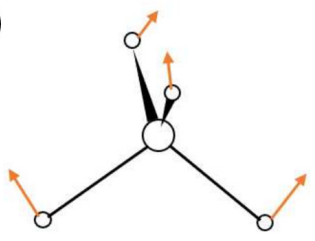

FIG. 4. Classical molecular dynamics simulation.(a) Normalized population of each breakup channel of $\mathrm{CH}_{4}^{+}$trajectories.(b) $f_{2}$ stretching mode can lead to $C_{3 v}$ geometry and contribute to $\mathrm{CH}_{3}^{+}+\mathrm{H}^{+}$channel. (c)e bending mode, (d) $f_{2}$ stretching mode and (e) $f_{2}$ bending mode can lead to $D_{2 d}$ or $C_{2 v}$ geometry and contribute to $\mathrm{CH}_{2}^{+}+\mathrm{H}_{2}^{+}$channel.

₹ characteristic time of the $\mathrm{JT}$ distortion from the $C_{3 v}$ to $C_{2 v}$ geometries is almost 20 fs.

3

The $e$ bending mode results in $D_{2 d}$ geometry, while the $f_{2}$ mode leads to $C_{3 v}$ and $C_{2 v}$ geometry and the former is preferred [24. So the 5 fs peak for both channels occur when most $\mathrm{CH}_{4}^{+}$monocations locate at $C_{3 v}$ geometry and the rest $C_{2 v}$ or $D_{2 d}$ geometry within $\frac{1}{4}$ period of $f_{2}$ and $e$ vibration. From Fig. $4(\mathrm{~b}) \sim(\mathrm{e})$, only the $C_{3 v}$ geometry from $f_{2}$ stretching mode will contribute to $\mathrm{CH}_{3}^{+}+\mathrm{H}^{+}$channel, but both $f_{2}$ and $e$ modes contribute to the $\mathrm{CH}_{2}^{+}+\mathrm{H}_{2}^{+}$channel. The three vibrations keep phasing and dephasing periodically due to their commensurate frequencies $\left(3019 \mathrm{~cm}^{-1}, 1534 \mathrm{~cm}^{-1}\right.$ and $\left.1306 \mathrm{~cm}^{-1}\right)$, which result in the maximum of $29 \mathrm{fs}$ for the $\mathrm{CH}_{2}^{+}+\mathrm{H}_{2}^{+}$channel (see Supplementary Material for detailed analysis).

The 5 fs and 29 fs peaks for $\mathrm{CH}_{2}^{+}+\mathrm{H}_{2}^{+}$channel are in a good agreement with $C_{2 v}$ population in Fig. 3(e), so does the $17 \mathrm{fs}$ and $36 \mathrm{fs}$ peaks in $\mathrm{CH}_{3}^{+}+\mathrm{H}^{+}$with the $C_{3 v}$ population. Combining the knowledge from the time-resolved two- and three-body measurements, the evolution of 

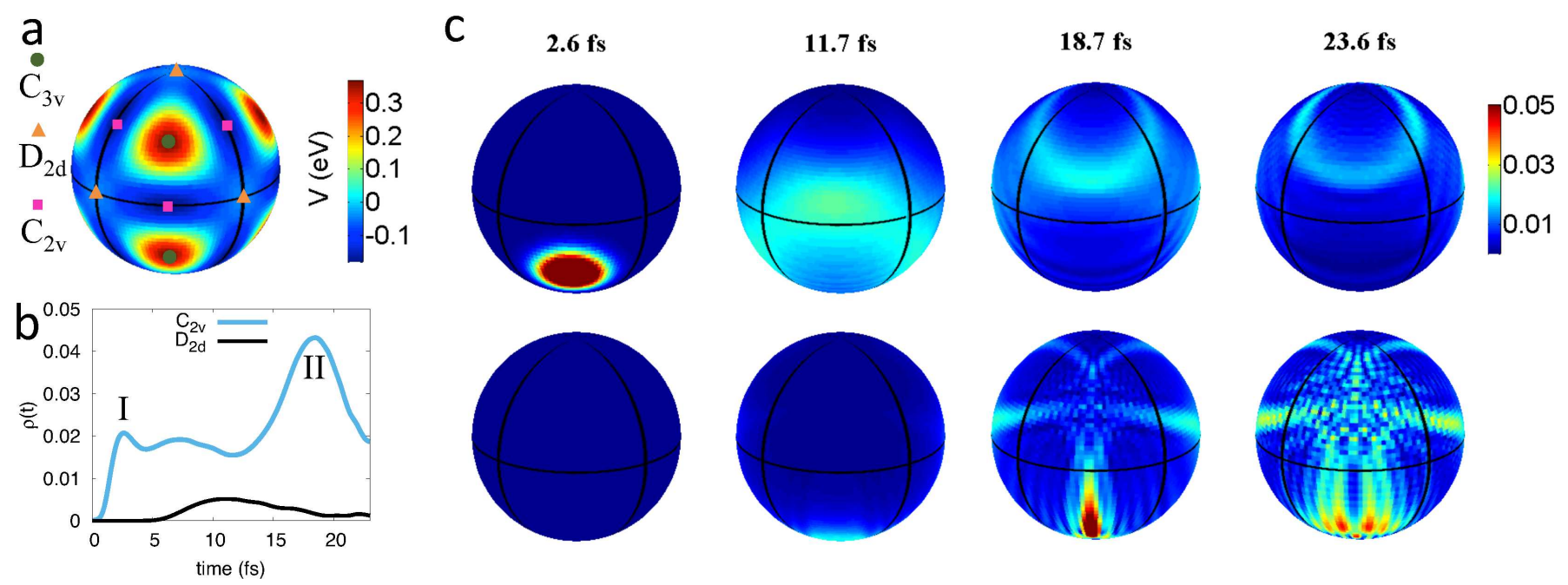

FIG. 5. Quantum wave packet dynamics simulation. (a) The potential energy surface of $\mathrm{CH}_{4}^{+}$cation in the sphere. (b) Time dependence of the populations in the $C_{2 v}$ and $D_{2 d}$ geometries of $\mathrm{CH}_{4}^{+}$cation. The results are obtained by initially putting the wavepacket on the $\mathrm{C}_{3 \mathrm{v}}$ geometry. I and II represents two distinct local maxima for the populations in the $C_{2 v}$ geometry. (c) Snapshots of wavepacket density $\rho(t)$ of $\mathrm{CH}_{4}^{+}$cation in the sphere starting from $\mathrm{C}_{3 \mathrm{v}}$ geometry. In (c), the top and bottom rows correspond to the front and back views of the sphere, respectively.

1 the molecular geometry has been directly imaged in real space. The excellent consistency of 2 the results extracted from two- and three-body data shows the accuracy and reliability of the direct imaging of $\mathrm{CH}_{4}^{+}$symmetry evolution.

For the three-body Coulomb explosion data in Fig. 3, we naturally classify the spot-like 5 structures as $S 1, S 2, S 3$ and map them to $D_{2 d}$-like, $C_{2 v}$-like and $C_{3 v}$-like configurations. its initial bond length and angle. $S 1$ has mixed contribution from $C_{3 v}$-like, $C_{2 v}$-like and $D_{2 d^{-}}$-like configurations. $S 2$ and $S 3$ are formed by contributions from $C_{2 v^{-}}$like and $D_{2 d^{-}}$like configurations, respectively. The measured Coulomb momentum map at a specific delay is modelled by the superposition of a set of Gaussian functions, with each set representing the related symmetric configuration (see Supplementary Material for detailed analysis). The result shown in Fig. 3(e) reveals two peaks at 5 fs and 29 fs for the $C_{2 v}$-like geometry, and another two peaks at 17 fs and 36 fs for the $C_{3 v^{-}}$like geometry. The time delay between the $C_{3 v}$-like and $C_{2 v}$-like geometries is consistent as that extracted from analysis of the two-body breakup channels shown in Fig. 2 . 
The analysis from the $a b$ initio classical MD can be refined with quantum wave packet dynamics simulation based on an effective Hamiltonian that maps the states involved in the $F \otimes(f \oplus e)$ JT effect of $\mathrm{CH}_{4}^{+}$onto the surface of a sphere [11, 25]. Each point represents a specific distorted geometry by $e$ and $f_{2}$ bending mode, as shown in Fig.55(a). The center of each face corresponds to the $C_{3 v}$ structure, which has the highest potential energy, while the center of each line segment corresponds to the $C_{2 v}$ structure, which has the lowest potential energy. The time dependence of the population in the $C_{2 v}$ and $D_{2 d}$ configurations starting from a single $C_{3 v}$ configuration is presented in Fig. 5(b) calculated by the quantum wave packet dynamics simulation. One can see that the wavepacket travels through an intermediate $D_{2 d}$ configuration, and the population of the $C_{2 v}$ configurations peaks at $\sim 18.7 \mathrm{fs}$, which is consistent with the time difference of the $\mathrm{CH}_{3}^{+}+\mathrm{H}^{+}$and $\mathrm{CH}_{2}^{+}+\mathrm{H}_{2}^{+}$peaks in the two-body Coulomb explosion.

The theory uncovers more delicate structure beyond the precision limit of experimental observations, that there are two distinct local maxima at the instants of $\sim 2.6 \mathrm{fs}$ and $\sim 18.7 \mathrm{fs}$ in the time-dependent population of the $C_{2 v}$ geometry (Fig. 5 (b)). To shed light on the origin of the two local maxima, we show in Fig. 5(c) the snapshots of wavepacket density $\rho(t)$ of $\mathrm{CH}_{4}^{+}$cation starting from the $C_{3 v}$ geometry. At $2.6 \mathrm{fs}$, the nuclear wave packet delocalizes and part of the wave packet distorts to the $C_{2 v}$ geometries, which give rise to the first local maximum in Fig. 5(b). This time scale is consistent with previous studies using high-order harmonic spectroscopy [18, 26 28]. However, this is not the only pathway in the formation of the $C_{2 v}$ geometry for the methane cation. The nuclear wave packet can continue to spread on the surface of the sphere because the potential barriers between the $C_{2 v}$ minima are so low, the $\mathrm{CH}_{4}^{+}$wavepacket can undergo a large-amplitude motion[10, 12]. The nuclear wave packet starts to rephase at the back side of the sphere, and form a maximum in the $C_{2 v}$ geometry at the back side of the sphere at $18.7 \mathrm{fs}$, which corresponds to the second local maximum in Fig. 5(b). The interference fringes of wave packet on the sphere in Fig. 5(c) shows consistency with the physical picture of phasing and dephasing dynamics revealed in the molecular dynamics simulation.

In summary, we have directly measured the ultrafast structural dynamics in real space during the onset of Jahn-Teller effect, and obtained the time, during which the JT drives the $\mathrm{CH}_{4}^{+}$cation to deform from the higher symmetric $C_{3 v}$ down to the $C_{2 v}$ configurations, to be $\sim 20 \mathrm{fs}$. We show that the nuclear wave packets of the methane cation experiences 
complex multimode phasing dynamics to reach the $C_{2 v}$ configurations. The interference among symmetric modes play a significant role in the formation of the $C_{2 v}$ geometry during the JT distortion process. This has a broad impact on the understanding of the structural rearrangement triggered by the strong laser pulses for the floppy molecular systems. Due to the universality of the JT effect, our study offers a possibility to obtain experimental information of detailed structural features and intramolecular dynamics of the floppy molecules and reveal the symmetry breaking dynamics in ultrashort time scale.

\section{Methods}

Experimental Methods. The experiment was performed using linearly polarized radiation from a Ti:sapphire laser system at 800-nm central wavelength with $\sim 25$ fs pulse width (FWHM). The laser pulse was split in a Mach-Zehnder type interferometer providing two nearly identical pulses separated by a time delay, which can be controlled by a motorized translation stage. The resulting two pulses were recombined before the vacuum chamber by using a beam combiner. The intensity of each laser pulse is estimated to be almost $3 \times 10^{14} \mathrm{~W} / \mathrm{cm}^{2}$. The laser beam was then focused into the vacuum chamber and interacted with the supersonic $\mathrm{CH}_{4}$ molecules. We measured the three-dimensional momentum distributions of the ions using cold-target recoil-ion momentum spectroscopy (COLTRIMS) 29].

Classical MD Simulation. To simulate the experiment, a set of trajectories of the $\mathrm{CH}_{4}^{+}$ cation is integrated up to 100 fs after the photo-ionization process. For each set of trajectories, $\mathrm{CH}_{4}^{+}$is promoted to $\mathrm{CH}_{4}^{2+} \mathrm{PES}$ at selected time-delays and the corresponding dissociation to either Coulomb explosion channel determined by following the subsequent trajectory (see Supplementary Material).

Quantum Wave Packet Dynamics Simulation. To understand the dynamics of the JT distortion, we carried out wave packet dynamics simulation using the multi-configuration timedependent Hartree (MCTDH) method [30, based on an effective Hamiltonian by considering molecular symmetry. In the Hamiltonian, the potential of the corresponding $F \otimes(f \oplus e)$ JT effect of $\mathrm{CH}_{4}^{+}$is mapped onto the surface of a sphere [11, 25], where the $e$ and $f_{2}$ vibrational modes are parametrized by spherical harmonics of order $l=2$. The details of the simulation is explained in the Supplementary Material. 


\section{Acknowledgements}

This work is supported by the National Natural Science Foundation of China (Grant Nos. 11722432, 11674116, and 61475055) and National Key Research and Development Program of China (Grant No. 2019YFA0308300). Z.L. and M.Z. are grateful to Yajiang Hao for helpful discussions.

\section{Author contribution}

M.L., K.G., Q.S., W.C., S.L., J.Q., Y.Z., Y.L., and P.L. designed the experiment and carried our the measurement. M.Z., O.V., Z.G., Q.Z., X.G., L.C., and Z.L. performed the classical and quantum molecular dynamics simulations. M.L., M.Z., O.V., and Z.L. prepared the manuscript. All authors contributed to finalizing and approving the manuscript.

M.L., M.Z. contributed equally to this work.

\section{Additional information}

Supplementary Information accompanies this paper.

Competing financial interests: The authors declare no competing financial interests.

\footnotetext{
* weicao@hust.edu.cn

$\dagger$ yunquan.liu@pku.edu.cn

$\ddagger$ zheng.li@pku.edu.cn

$\S$ lupeixiang@hust.edu.cn
}

[1] H. A. Jahn and E. Teller, Proc. R. Soc. Lond. A 161, 220 (1937).

[2] J. Yang, X. Zhu, T. J. A. Wolf, Z. Li, J. P. F. Nunes, R. Coffee, J. P. Cryan, M. Gühr, K. Hegazy, T. F. Heinz, K. Jobe, R. Li, X. Shen, T. Veccione, S. Weathersby, K. J. Wilkin, C. Yoneda, Q. Zheng, T. J. Martinez, M. Centurion, and X. Wang, Science 361, 64 (2018).

[3] P. W. Higgs, Phys. Rev. Lett. 13, 508 (1964).

[4] H. von Busch, V. Dev, H.-A. Eckel, S. Kasahara, J. Wang, W. Demtröder, P. Sebald, and W. Meyer, Phys. Rev. Lett. 81, 4584 (1998) 
$1 \quad$ [5] R. Meiswinkel and H. Köppel, Chem. Phys. 144, 117 (1990).

2 [6] W. E. Ernst and S. Rakowsky, Phys. Rev. Lett. 74, 58 (1995).

3 [ [7] L. B. Knight, J. Steadman, D. Feller, and E. R. Davidson, J. Am. Chem. Soc. 106, 3700

4 (1984).

${ }_{5}$ [ [8] L. B. Knight, G. M. King, J. T. Petty, M. Matsushita, T. Momose, and T. Shida, J. Chem.

$6 \quad$ Phys. 103, 3377 (1995).

7 [ [9] R. Lindner, K. Müller-Dethlefs, E. Wedum, K. Haber, and E. R. Grant, Science 271, 1698 $8 \quad(1996)$.

9 [10] H. J. Wörner, X. Qian, and F. Merkt, J. Chem. Phys. 126, 144305 (2007).

10 [11] U. Jacovella, H. J. Wörner, and F. Merkt, J. Mol. Struct. 343, 62 (2018).

[12] H. J. Wörner, R. van der Veen, and F. Merkt, Phys. Rev. Lett. 97, 173003 (2006).

[13] H. Wörner and F. Merkt, Angew. Chem. Int. Ed. 48, 6404 (2009).

[14] S. Sen and T. Mondal, Mol. Phys. 117, 2487 (2019).

[15] Z. Vager, E. P. Kanter, G. Both, P. J. Cooney, A. Faibis, W. Koenig, B. J. Zabransky, and D. Zajfman, Phys. Rev. Lett. 57, 2793 (1986).

[16] Z. Vager, T. Graber, E. P. Kanter, and D. Zajfman, Phys. Rev. Lett. 70, 3549 (1993).

[17] J. Yang, X. Zhu, T. J. Wolf, Z. Li, J. P. F. Nunes, R. Coffee, J. P. Cryan, M. Gühr, K. Hegazy, T. F. Heinz, et al., Science 361, 64 (2018).

[18] S. Baker, J. S. Robinson, C. A. Haworth, H. Teng, R. A. Smith, C. C. Chirila, M. Lein, J. W. G. Tisch, and J. P. Marangos, Science 312, 5 (2006).

[19] D. Schaniel, M. Nicoul, and T. Woike, Phys. Chem. Chem. Phys. 12, 9029 (2010).

[20] Y. Pertot, C. Schmidt, M. Matthews, A. Chauvet, M. Huppert, V. Svoboda, A. Von Conta, A. Tehlar, D. Baykusheva, J.-P. Wolf, et al., Science 355, 264 (2017).

[21] R. F. Frey and E. R. Davidson, J. Chem. Phys. 88, 1775 (1988).

[22] I. A. Bocharova, A. S. Alnaser, U. Thumm, T. Niederhausen, D. Ray, C. L. Cocke, and I. V. Litvinyuk, Phys. Rev. A 83, 013417 (2011).

[23] S. Koseki, N. Shimakura, Y. Teranishi, S. H. Lin, and Y. Fujimura, J. Phys. Chem. A 117, $333(2013)$.

[24] I. B.Bersuker, The Jahn-Teller Effect (Cambridge University Press, 2006).

[25] C. Chancey, M. O'Brien, and M. O'Brien, The Jahn-Teller effect in $\mathrm{C}_{60}$ and other icosahedral complexes (Princeton University Press, 1997). 
1 [26] C. B. Madsen, M. Abusamha, and L. B. Madsen, Phys. Rev. A 81, 043413 (2010),

2 [27] T. Mondal and A. J. C. Varandas, J. Chem. Theo. Comput. 10, 3606 (2014),

3 [28] T. Mondal and A. J. C. Varandas, J. Chem. Phys. 143, 014304 (2015).

4 [29] J. Ullrich, R. Moshammer, A. Dorn, R. Dörner, L. Schmidt, and H. Schmidt-Böcking, Struc-

$5 \quad$ ture and Bonding 71, 1463 (2003).

6 [30] M. H. Beck, A. Jäckle, G. A. Worth, and H.-D. Meyer, Phys. Rep. 324, 1 (2000). 


\section{Figures}

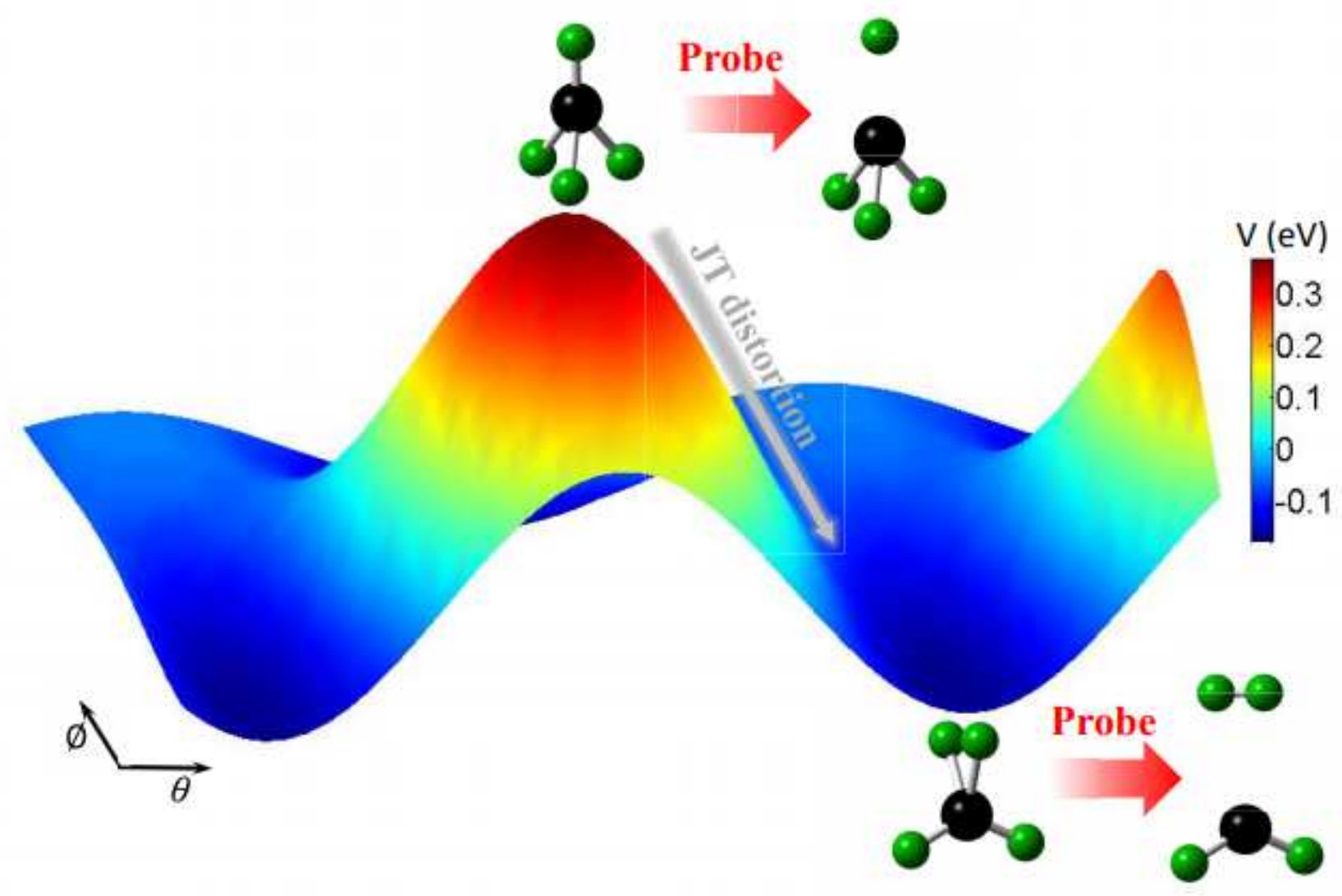

Figure 1

Scheme of probing JT deformation in a photoionized $\mathrm{CH}+4$ cation. The $\mathrm{CH}+4$ cation in a $\mathrm{C} 3 \mathrm{v}$ geometry (at the peak of the potential energy surface) undergoes JT distortion to a C2v geometry (at the bottom of the potential energy surface). By recording the coincident fragments from Coulomb explosion of those geometries as a function of time between the pumpprobe laser pulses, the dynamics of the JT deformation can be revealed. 

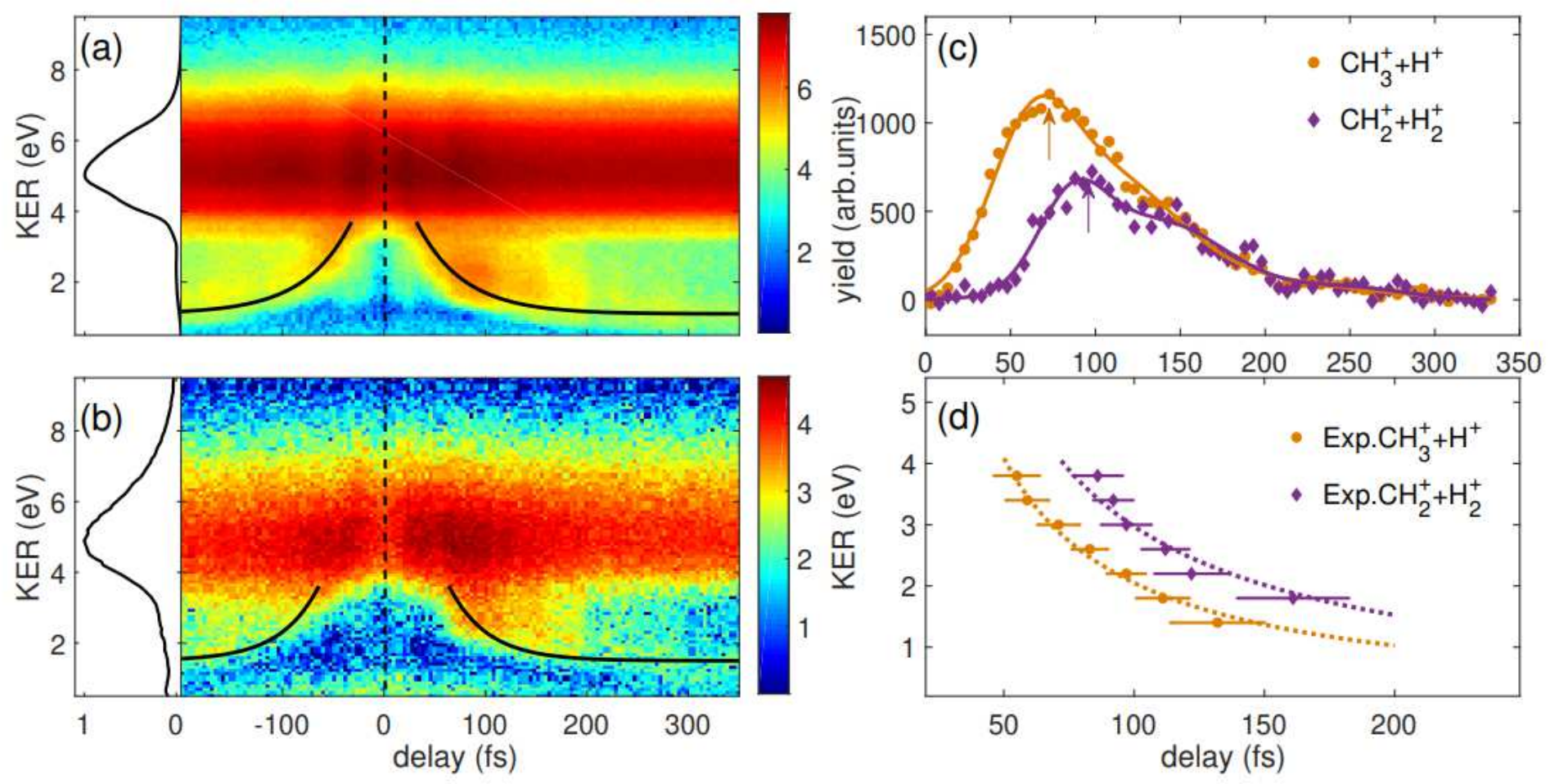

\section{Figure 2}

Time evolution of two-body breakup channel. (a)(b) The measured ion yields with respect to the KER and the pump-probe time delay for the pathways of $\mathrm{CH}+3+\mathrm{H}+(\mathrm{a})$ and $\mathrm{CH}+2+\mathrm{H}+2$ (b). The time-integrated KER distributions are shown in the left panels. The black solid curves in (a) and (b) are used to guide the time-dependent feature. (c) The measured ion yields with respect to the time delay at the KER of $3.0 \mathrm{eV}$ for the pathways of $\mathrm{CH}+3+\mathrm{H}+$ and $\mathrm{CH}+2+\mathrm{H}+2$ (multiplied by a factor of 5 for visual convenience). The arrows show the peaks of the time-dependent distributions. (d) The peak extracted from the measured time-dependent distribution for different KERs. The dotted lines are the fits of the experimental data. 

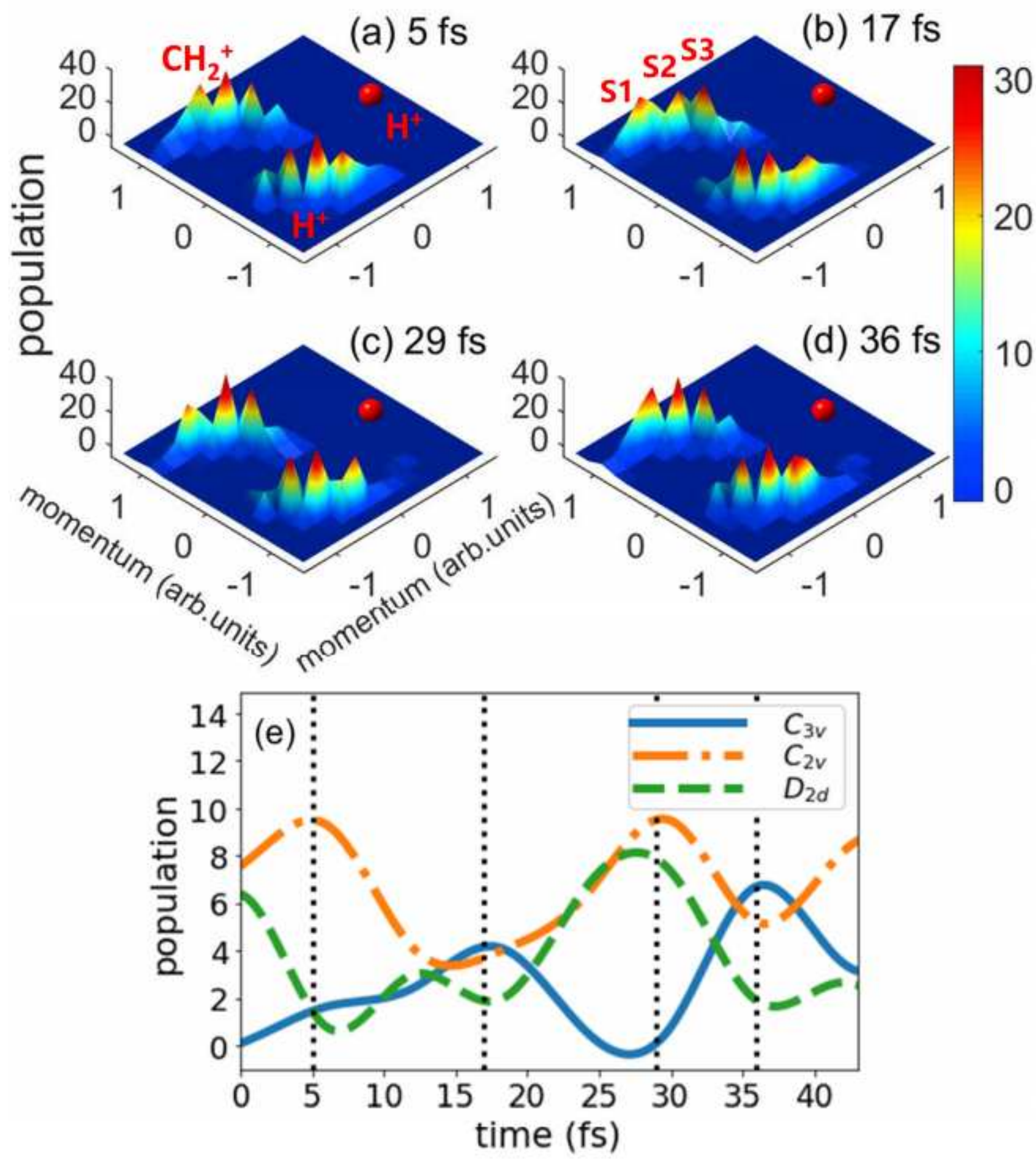

Figure 3

Time evolution of three-body breakup channel. (a)-(d) Newton plot at the delay of $5 \mathrm{fs}, 17 \mathrm{fs}, 29 \mathrm{fs}$, and 36 fs. Several spot-like structures in the Newton plot are indicated by S1, S2, and S3 in (b), which represent molecular geometries of different symmetry types at the instant of Coulomb explosion. The momentum vector of the first $\mathrm{H}+$ is set as one unit at $(0,1)$, as shown by the red sphere. The momentum vectors of the $\mathrm{CH}+2$ ion and the second $\mathrm{H}+$ ion are normalized with respect to the length of momentum vector of 
the first $\mathrm{H}+$ ion. (e) The experimentally extracted evolution of C3v like, C2v like and D2d like geometries. The experimental data are obtained by selecting those events with the same final momenta for two $\mathrm{H}+$.

(a)

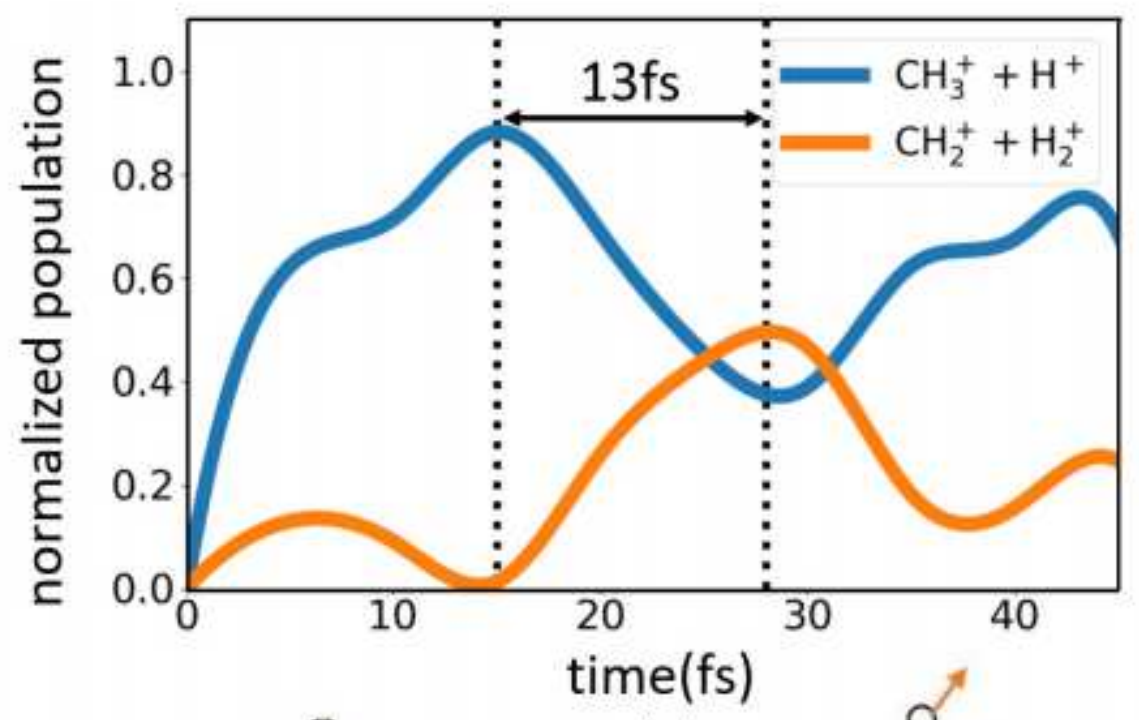

(b)

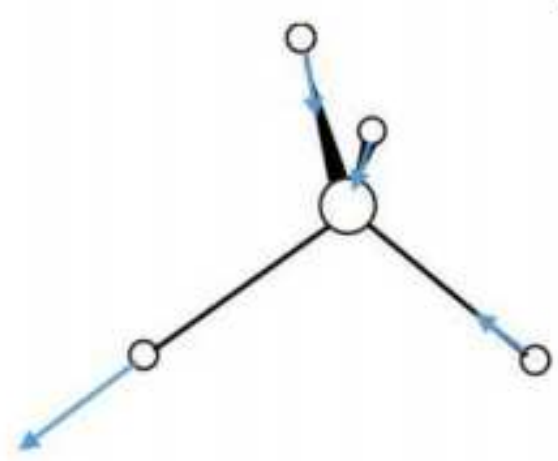

(c)

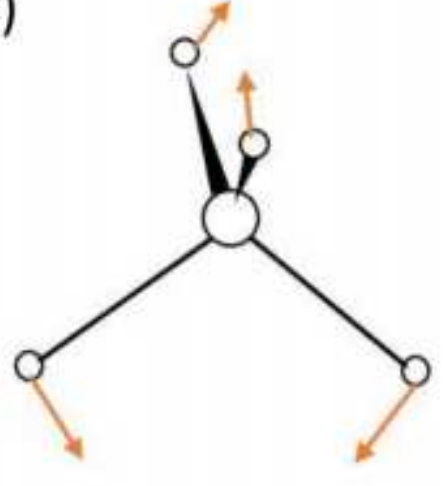

(d)

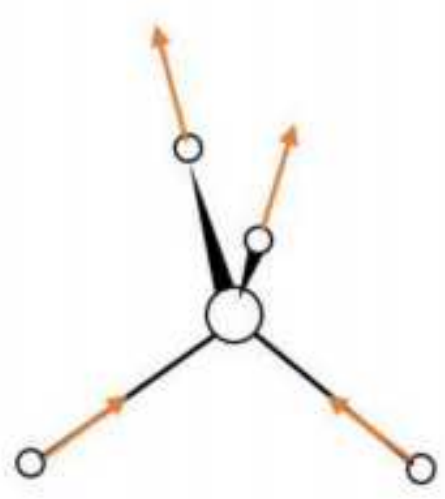

(e)

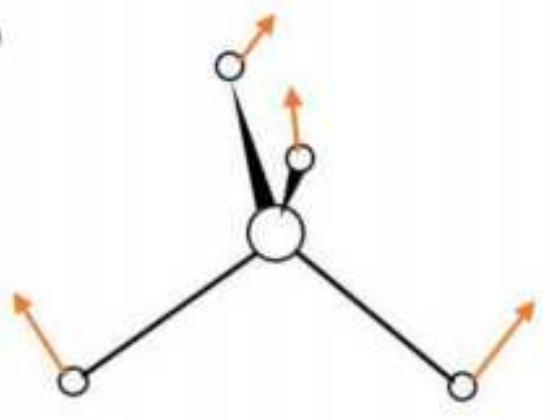

Figure 4

Classical molecular dynamics simulation.(a) Normalized population of each breakup channel of $\mathrm{CH}+4$ trajectories.(b) f2 stretching mode can lead to $\mathrm{C} 3 v$ geometry and contribute to $\mathrm{CH}+3+\mathrm{H}+$ channel. (c)e bending mode, (d) f2 stretching mode and (e) f2 bending mode can lead to D2d or C2v geometry and contribute to $\mathrm{CH}+2+\mathrm{H}+2$ channel. 

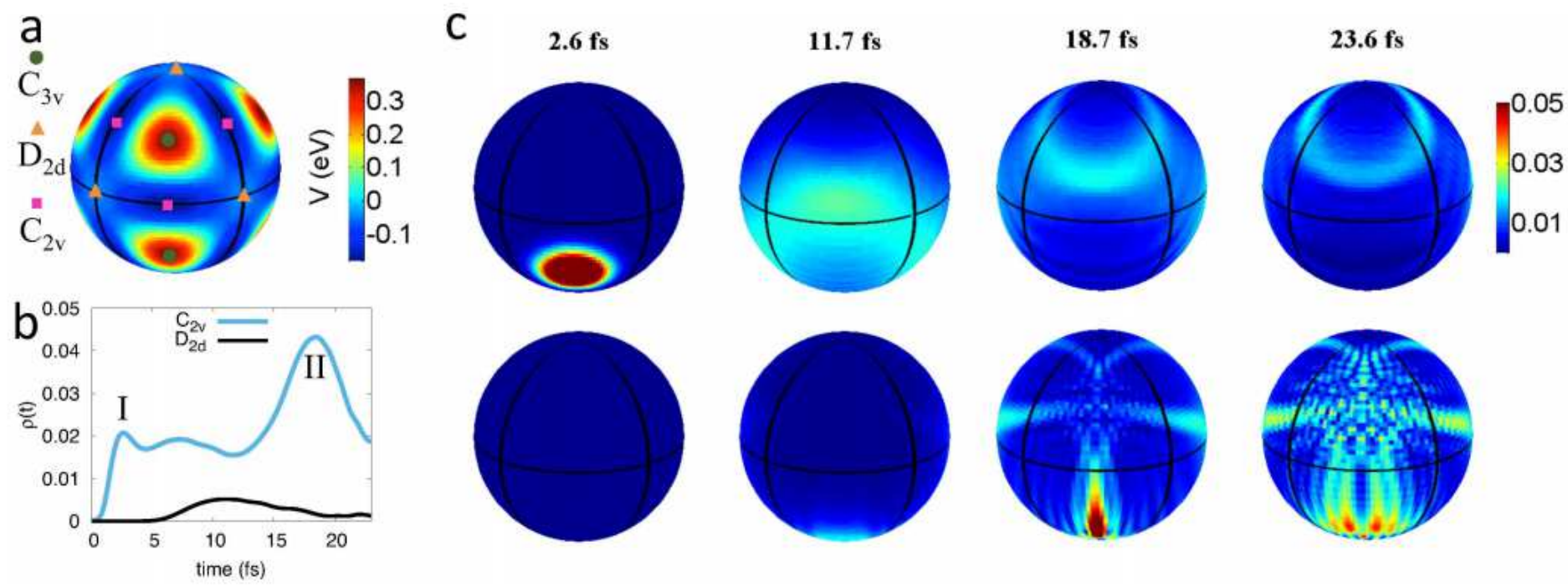

\section{Figure 5}

Quantum wave packet dynamics simulation. (a) The potential energy surface of $\mathrm{CH}+4$ cation in the sphere. (b) Time dependence of the populations in the $\mathrm{C} 2 \mathrm{v}$ and $\mathrm{D} 2 \mathrm{~d}$ geometries of $\mathrm{CH}+4$ cation. The results are obtained by initially putting the wavepacket on the C3v geometry. I and II represents two distinct local maxima for the populations in the $\mathrm{C} 2 \mathrm{v}$ geometry. (c) Snapshots of wavepacket density $\rho(t)$ of $\mathrm{CH}+4$ cation in the sphere starting from $\mathrm{C} 3 \mathrm{v}$ geometry. In (c), the top and bottom rows correspond to the front and back views of the sphere, respectively.

\section{Supplementary Files}

This is a list of supplementary files associated with this preprint. Click to download.

- suppl.zip 